Bulletin of the Seismological Society of America. Vol. 61, No. 5, pp. 1201-1211. October, 1971

\title{
ZERO BASELINE CORRECTION OF STRONG-MOTION ACCELEROGRAMS
}

\author{
By Mihailo D. Trifunac*
}

\begin{abstract}
A new method is proposed for standard baseline correction of strong-motion accelerograms. It is based on high-pass filtering of the uncorrected digitized accelerogram data. Unlike the parabolic baseline correction, the new method has well-defined frequency transfer function properties which are largely independent of the record length.
\end{abstract}

\section{INTRODUCTION}

A detailed quantitative knowledge of the nature of ground motion close to the source of an earthquake release comes from strong-motion accelerographs (e.g., see Hudson, 1963; 1965; Fremd, 1963; Halverson, 1965; 1970; Cloud, 1964; and Trifunac and Hudson, 1970). Several hundreds of such instruments are presently installed in the western United States and are maintained by the Seismological Field Survey of the National Ocean Survey (NOS formerly USCGS). The only other comparable network has evolved during the last 15 years in Japan.

Since the first strong-motion accelerogram was obtained some 40 years ago during the 1933 Long Beach, California earthquake, more than 100 strong-motion accelerograms have been recorded that are of special value for earthquake engineering and strong-motion seismology research. In order to extract maximum information from these records, optimum methods for accelerograph data digitizing and processing must be developed. In this paper, we consider zero baseline correction procedures.

An accelerograph transducer, usually a single-degree-of-freedom, 60 per cent damped oscillator with a natural frequency of $12 \mathrm{cps}$ to $25 \mathrm{cps}$ is used to record ground acceleration. The recorded instrument response approximates ground acceleration up to about 5 cps to $10 \mathrm{cps}$. With only a few exceptions (Jenschke and Penzien, 1964; McLennan, 1969; and Trifunac and Hudson, 1970), the accelerograph record is usually taken to represent actual ground acceleration and no instrument correction is applied to it. For calculations of ground velocity and displacement, which are smoothing processes, accelerogram records do not have to be corrected for instrument response.

A digitized accelerogram cannot be integrated immediately in an attempt to determine ground velocity and displacement for the following reasons. The initial velocity and displacement, and the actual zero base line for the digitized accelerogram are not known. Of these three unknowns, finding the zero base line of the accelerogram is the most important step in accelerogram data processing.

There have been many contributions to the general problem of digitization, baseline correction, and double integration of the accelerogram. Some of these investigations were by McComb, Ruge and Neumann, 1943; Housner, 1947; Hershberger, 1955; Berg and Housner, 1961; Berg, 1963; Amin and Ang, 1966; Brady, 1966; Schiff and Bogdanoff, 1967; Poppitz, 1968; Hudson, Nigam and Trifunae, 1969; and Boyce, 1970.

A majority of these investigators assume the zero acceleration base line to be of the parabolic form. Although no physical justification may be given for a curve of higher

\footnotetext{
* Present address : Lamont-Doherty Geological Observatory of Columbia University, Palisades, New York, 10964.
} 
degree than the first, a straight line (Hudson et al., 1969), it has been generally agreed that the errors in the digitized accelerogram caused by the warping of the record and other similar distortions are in most cases corrected by the parabolic base line. Also, it has been shown (Hudson et al., 1969) that the spurious periods introduced in the accelerogram by the parabolic baseline correction do not seriously affect the accuracy of the response spectra calculations for periods up to about 5 sec. The effects of the parabolic baseline correction on the ground velocity and displacement of relatively short record durations, assuming zero initial conditions, have been found to be relatively minor (Hudson et al., 1969). For intermediate and long accelerograms, however, a parabolic base line is not adequate. This is because errors in ground displacement introduced by digitization, warping, and the transverse play of the accelerograph paper in an instrument mainly affect intermediate and long periods. Also, the parabolic baseline correction does not meet all of the requirements for the standard baseline correction, because it depends largely on the record length, and the low-frequency components will be treated differently from one accelerogram to another.

At the present time, an increasing number of high buildings with long fundamental periods of vibration and studies related to the nature of the earthquake energy release require that the accuracy of the digitized accelerograms be extended to periods for which parabolic baseline correction is not adequate.

\section{Accelerogram Baseline Correction}

The criteria for a baseline correction of the strong-motion accelerogram can be clearly formulated from the physical point of view as follows. The baseline-corrected digitized accelerogram data should be processed in such a way that most of the actual physically real signals are preserved while the long-period noise introduced into the data by the digitization process is eliminated. It would be easy to achieve the separation of the long-period digitization errors if they were represented by a well-defined frequency band. Although sharp boundaries cannot be unequivocally determined, the analysis of the long-period digitization errors (Trifunac, 1970) shows that for periods longer than about 16 sec, digitization noise may give excessively large displacement amplitudes. In the decision process of determining this cutoff period, our present preference would be to filter out some of the possibly realistic seismic signals rather than to contaminate the baseline-corrected data with processing errors. The final choice of the cutoff period of $16 \mathrm{sec}$ (Trifunac, 1970) is a consequence of the presently available digitizing equipment at the California Institute of Technology and the accuracy of the operators who are currently digitizing accelerograph records. The advancement of digitizing technology will, no doubt, change data processing capabilities and the present limits will be changed accordingly.

We now describe the details of zero baseline correction procedure which is briefly summarized in the flow chart in Figure 1. Prior to filtering, a straight zero acceleration baseline is least-square fitted to the uncorrected accelerogram $a\left(t_{i}\right)$. This has the effect of slightly rotating the base line to correct for any lack of parallelism between film and print in the enlargement process. Although not essential, this step is expected to improve the accuracy of the filtering procedures later applied to the accelerogram $a_{2}(n \Delta t)$. This step should actually be applied only to records enlarged from $35 \mathrm{~mm}$ and $70 \mathrm{~mm}$ negatives and is not required for directly-recorded paper records. Since for the paper recording instruments this rotation would, in any event, be small compared to the effects of the filtering process, it has been decided to apply this step uniformly to all digitized uncorrected data prior to the filtering, irrespective of the type of the original record. 
Before filtering, still another correction is made. It consists of fitting a straight line to the velocity obtained by integrating the $a_{1}{ }^{\circ}\left(t_{i}\right)$ accelerogram. The linear term in this correction introduces a constant $v_{1}$ translation in the base line of $a_{1}^{\circ}\left(t_{i}\right)$. The purpose of this correction is described later.

Since digital filtering requires equally-spaced data, 50 points per second are next interpolated to the unequally-digitized accelerogram $a_{1}\left(t_{i}\right)$ to give $a_{2}(n \Delta t)$, with $\Delta t=$ 0.02 sec. The removal of long periods is performed by low-pass filtering of the accelerogram by an Ormsby digital filter (Ormsby, 1961) and then subtracting the loss-pass filtered accelerogram (equivalent to a new zero base line) from the original accelero-

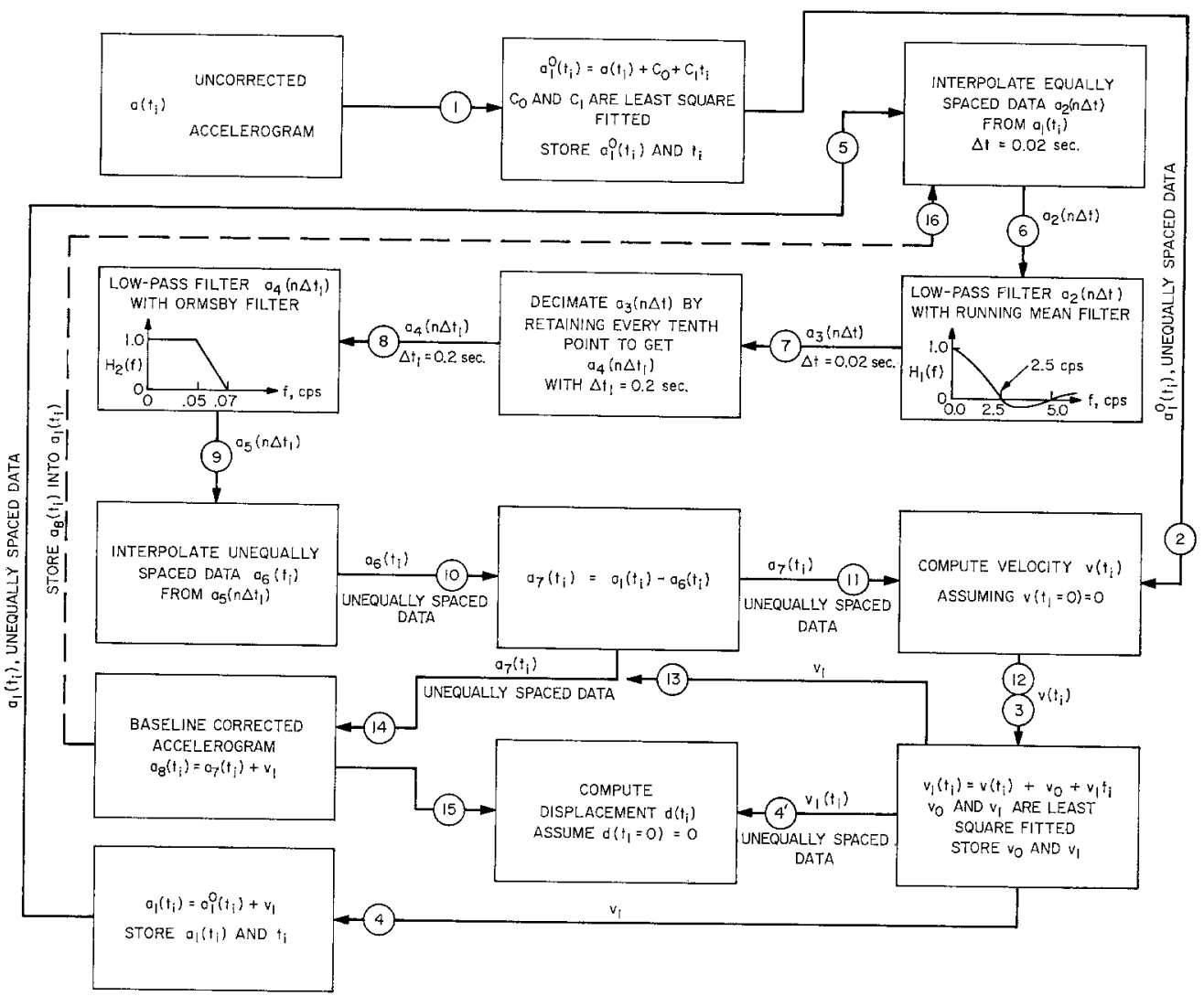

Fig. 1. Flow chart for baseline correction.

gram. As is well known, direct application of the Ormsby filter to the acceleration data would not be economical, since for cutoff periods of $16 \mathrm{sec}$, the filtering window must be as long as the whole accelerogram or even longer. To accomplish more economical filtering, we first low-pass filter the accelerogram using the equally-weighted running mean filter with a window width $T_{w}=0.40 \mathrm{sec}$. The transfer function $H_{1}(f)$ of such a digital filter is given approximately by (Holloway, 1958),

$$
H_{1}(f)=\frac{\sin \left(\pi f T_{w}\right)}{\pi f T_{w}}
$$

For $f=0$ we have $H_{1}(f)=1$ and for an increasing frequency $H_{1}(f)$ decreases toward 
its first zero at $f T_{w}=1$. For $T_{w}=0.40$ sec., $f=2.5$ cps. It is easy to show that this filter does not introduce any phase shifts into the original unfiltered data.

After filtering, the low-pass filtered data $a_{3}(n \Delta t)$ (Figure 1) are decimated and only every tenth point is kept. The new $\Delta t$ thus becomes 0.20 sec. The new data have the Nyquist frequency $f_{N}=2.5 \mathrm{cps}$, and preserve all of the long-period components up to about $f=0.10$ cps with essentially undistorted amplitudes. This is shown in Figure 2 in which $H_{1}(f)$ is plotted for low frequencies up to $f=0.10 \mathrm{cps}$. The low-pass filtered accelerogram data $a_{4}\left(n \Delta t_{1}\right)$ are now used as an input to a low-pass Ormsby filter. The result will be $a_{5}\left(n \Delta t_{1}\right)$ (Figure 1$)$.

Following Ormsby (1961), we define $\lambda=\omega / \omega_{S}, \lambda_{C}=\omega_{C} / \omega_{S}$ and $\lambda_{R}=\left(\omega_{T}-\omega_{C}\right) /$ $\omega_{S}$, where $\omega_{C}=$ cutoff frequency $\left(2 \pi f_{C}\right)$ and $\omega_{T}=$ filter roll-off termination frequency. The roll-off from $\omega_{C}$ to $\omega_{T}$ is linear. With time coordinate $t_{n}=n \Delta t_{1}$ and $\Delta t_{1}=1 / f_{S}$,

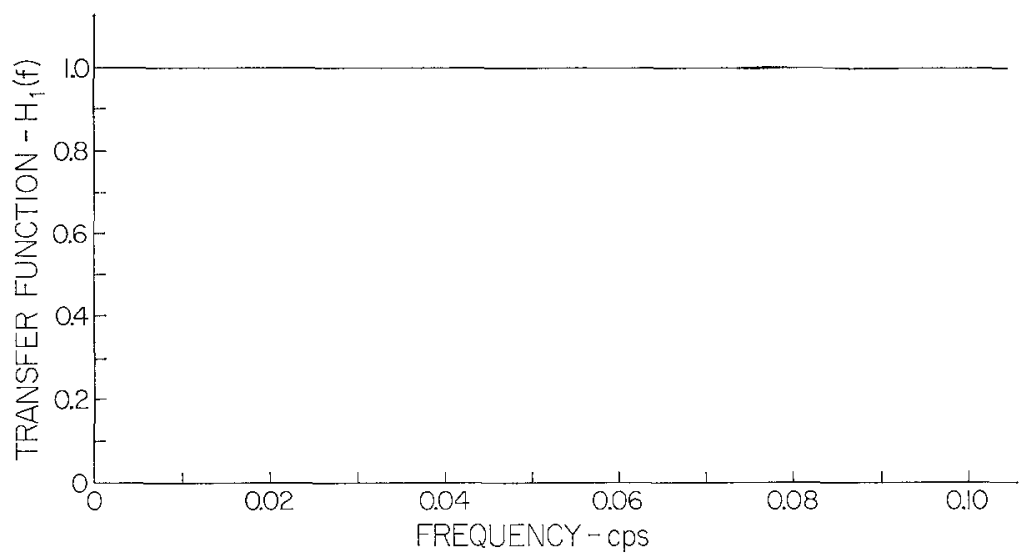

FIG. 2. Transfer function $H_{1}(f)$ for equally-weighted running mean filter with averaging interval $T_{w}=0.40 \mathrm{sec}$.

the weights for the Ormsby filter become

$$
h_{n}=\frac{\cos 2 \pi n \lambda_{C}-\cos 2 \pi n \lambda_{T}}{2 \lambda_{R}(\pi n)^{2}} ; \quad\left\{\begin{array}{l}
n=0, \pm 1, \pm 2, \cdots, \pm N \\
\lambda_{T}=\lambda_{C}+\lambda_{R}
\end{array}\right.
$$

The quantity $\lambda_{R}=\left(\omega_{T}-\omega_{C}\right) / \omega_{S}$ specifies the sharpness of roll-off after $\lambda_{C}$ and together with the finite number of weights $N$ measures the resultant accuracy of the filter. The accuracy is reduced for lower $\lambda_{R}$ and/or lower $N$. For $N \rightarrow \infty$, the transfer function $H_{2}(f)$ approaches the ideal $\hat{H}_{2}(f)$ given by

$$
\hat{H}_{2}(f)= \begin{cases}1 \quad ; & f \leqq f_{C} \\ \frac{f_{T}-f}{f_{T}-f_{C}} ; & f_{C} \leqq f \leqq f_{T} \\ 0 \quad ; & f>f_{C} .\end{cases}
$$

The dependence of the error

$$
\epsilon(f, N)=H_{2}(f)-\hat{H}_{2}(f)
$$


on $\lambda_{R}$ and $N$ is given by Ormsby (1961) as

$$
\epsilon \approx \frac{0.012}{\lambda_{R}} N
$$

This expression is independent of $\lambda_{G}$ and $f$ and is adopted in the computer program for the baseline correction to give $N$ and, thus, the width of the filtering window for the given $\lambda_{R}$ such that $\epsilon<1.2$ per cent. The computed transfer function $H_{2}(f)$ based on

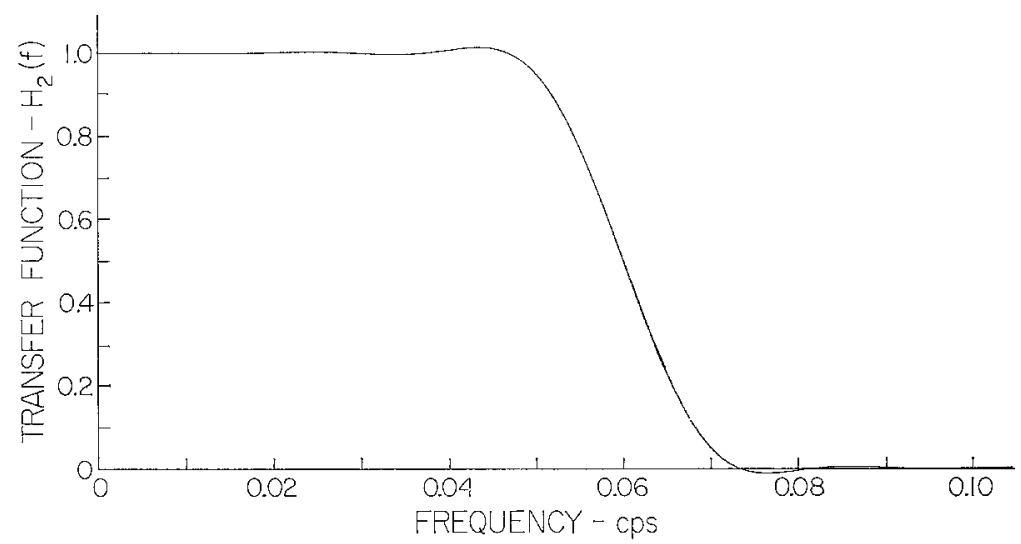

FIG. 3. Transfer function $H_{2}(f)$ for Ormsby filter $\left(f_{C}=0.05 \mathrm{eps}, f_{T}=0.07 \mathrm{cps}\right)$ with 250 weights over the filtering interval $T_{w}=100 \mathrm{sec}$.

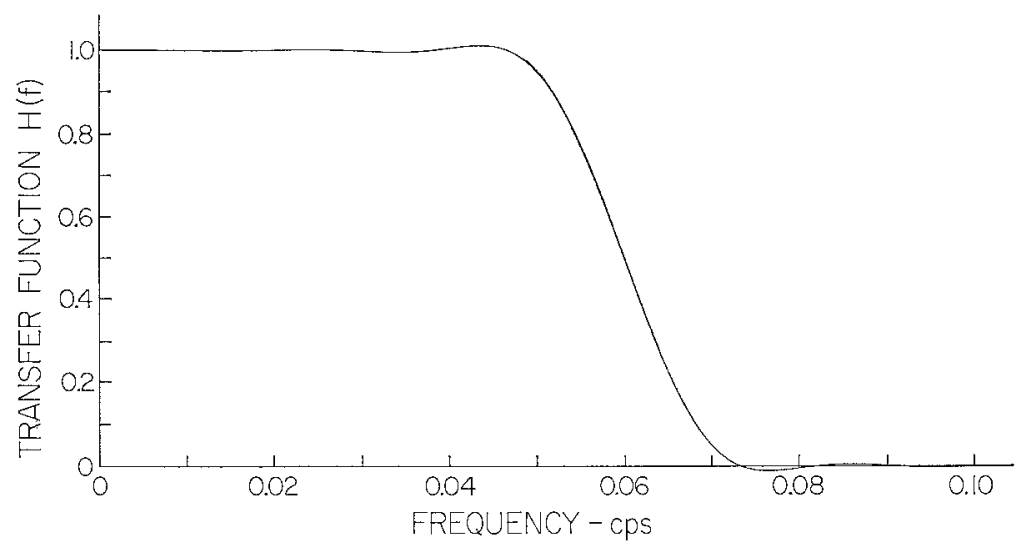

Fig. 4. Transfer function $H(f)=H_{1}(f) H_{2}(f)$ (See Figures 2 and 3).

the $N=250$ filter weights for $\lambda_{R}=0.004$ is plotted in Figure 3. The resultant transfer function $H(f)$ of the combined running mean filter and Ormsby filter is then given by the product of the transfer functions $H_{1}(f)$ and $H_{2}(f)$ and is plotted in Figure 4 . Since for the frequencies considered $H_{1}(f)$ is nearly unity, the resulting transfer function in Figure 4 is almost the same as that in Figure 3 for $H_{2}(f)$. The resulting low-pass filtered data $a_{5}\left(n \Delta t_{1}\right)$ now represents the zero base line apart from an additive constant.

The preliminary baseline-corrected accelerogram $a_{7}\left(t_{i}\right)$ is next obtained by subtracting the low-pass filtered accelerogram $a_{6}\left(t_{i}\right)$ from the original unequally-spaced data $a_{1}\left(t_{i}\right)$. When these data are integrated once to get the ground velocity and twice to get 
the ground displacement, it will be seen that two additional constants are required, namely, the initial velocity $v_{0}$ and the initial displacement $d_{0}$. The following simple physical reasons will indicate the best way to determine these constants.

As may be recalled, the baseline-corrected data $a_{7}\left(t_{i}\right)$ are obtained by subtracting the low-pass filtered accelerogram $a_{6}\left(t_{i}\right)$ from the accelerogram $a_{1}\left(t_{i}\right)$ from which a least-square-fitted straight line and a constant $v_{1}$ were subtracted, as described earlier. Although the slope of this straight line approximately eliminates distortions caused by photographic enlargement, the constant term $c_{0}$ in such a correction may well be in error because there is no physical reason for the mean value of the ground acceleration over the time interval considered to be zero. Since the mean value of the ground acceleration is actually very small, as an initial step, the uncorrected data are processed assuming that the mean of the acceleration is zero. It is further clear from the physical nature of the strong earthquake ground motion that for large time the velocity must tend to zero. From these considerations, it may be concluded that a straight-line error is present in the computed velocity and that under these conditions, it will not tend to zero for $t \rightarrow \infty$. A simple procedure is, then, to eliminate this error by fitting a twoparameter straight line $v_{0}+v_{1} t$ to the computed ground velocity. Such a procedure also gives an estimate of the initial velocity $v_{0}$. The correction $v_{1}$, the mean of the ground acceleration curve, applied before the filtering process introduces a d.c. change in the as yet uncorrected baseline accelerogram $a_{1}\left(t_{i}\right)$, and, thus, may require some small additional changes. We now consider reasons for these additional corrections.

The accelerogram to be analyzed is given in digitized form over the time interval from 0 to $T$ and is undefined outside that interval. If the time window over which filtering is performed is designated by $T_{w}$, then the accelerogram to be filtered must be extended from the 0 to $T$ interval to the $-T_{w} / 2$ to $T+T_{w} / 2$ interval. To specify the accelerogram outside the interval 0 to $T$, it may be assumed that the instrument was triggered by one of the first arrivals of earthquake waves so that a very short and hopefully insignificant portion of the accelerogram is lost, over a short interval of time, say $-\tau<t<0$. We assume that the accelerogram is zero in this time interval $-\tau<t<0$. Prior to any arrival of seismic waves, there is no motion, so the accelerogram is identically zero for $t<-\tau$. The time $T$, at the other end of the record, where digitization is stopped, is chosen by the operator and involves a certain amount of subjective judgment. The digitization is extended in time to the point where the accelerograph stopped recording, or where visually no "significant" signals can be detected on the accelerogram. It also may be necessary to stop digitization of the record at some earlier time $T$, because the size of the record exceeds the available room on the digitizer. For the purpose of the present analysis, we will make a crude but reasonable assumption that the accelerogram is zero for $t>T$. In this way, we have extended the accelerogram outside the interval 0 to $T$. The zero-extended accelerogram outside the interval 0 to $T$ is thus defined relative to the first straight-line baseline correction $c_{0}+c_{1}+v_{1}$ (steps 1,2,3 and 4 ; Figure 1 ) where constants $c_{0}, c_{1}$ and $v_{1}$ are determined prior to any filtering procedures. After the first low-pass filtering (steps 6, 7, . . etc., Figure 1), this zero level is changed through the change in $v_{1}$, and it may be necessary to correct the zero base line again for these additional boundary effects. The simplest way of carrying this correction out is to put the once-corrected accelerogram through the whole procedure several times more until the difference between the two baseline corrections is negligibly small. This iterative technique is indicated in the flow chart of Figure 1 with a dashed line.

To demonstrate how the above-described baseline correction method filters out long- 
period components in the accelerogram and ground displacement, we consider the N21E component of acceleration recorded in Taft, California, during the July 21, 1952 earthquake. Figure 5 gives a plot of this accelerogram (Hudson, Brady, Trifunac, 1969). We first least-square fit a straight sloping line to the accelerogram and then least-square fit a straight line on the velocity, which gives an estimate of the initial velocity $v_{0}$. By using this estimate of $v_{0}$ and double integrating $a_{1}\left(t_{i}\right)$, one obtains the ground displacement as shown in Figure 6. These procedures would correspond to steps 1, 2, 3

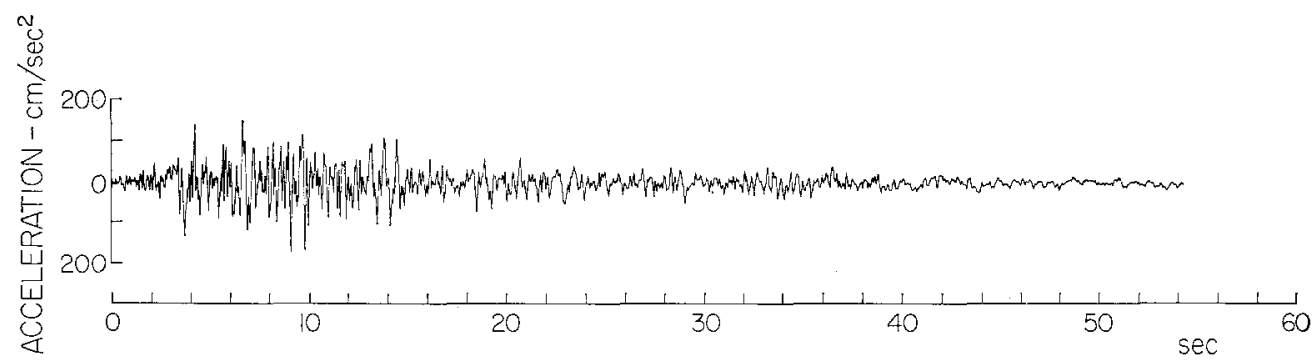

FIG. 5. Taft strong-motion accelerogram.

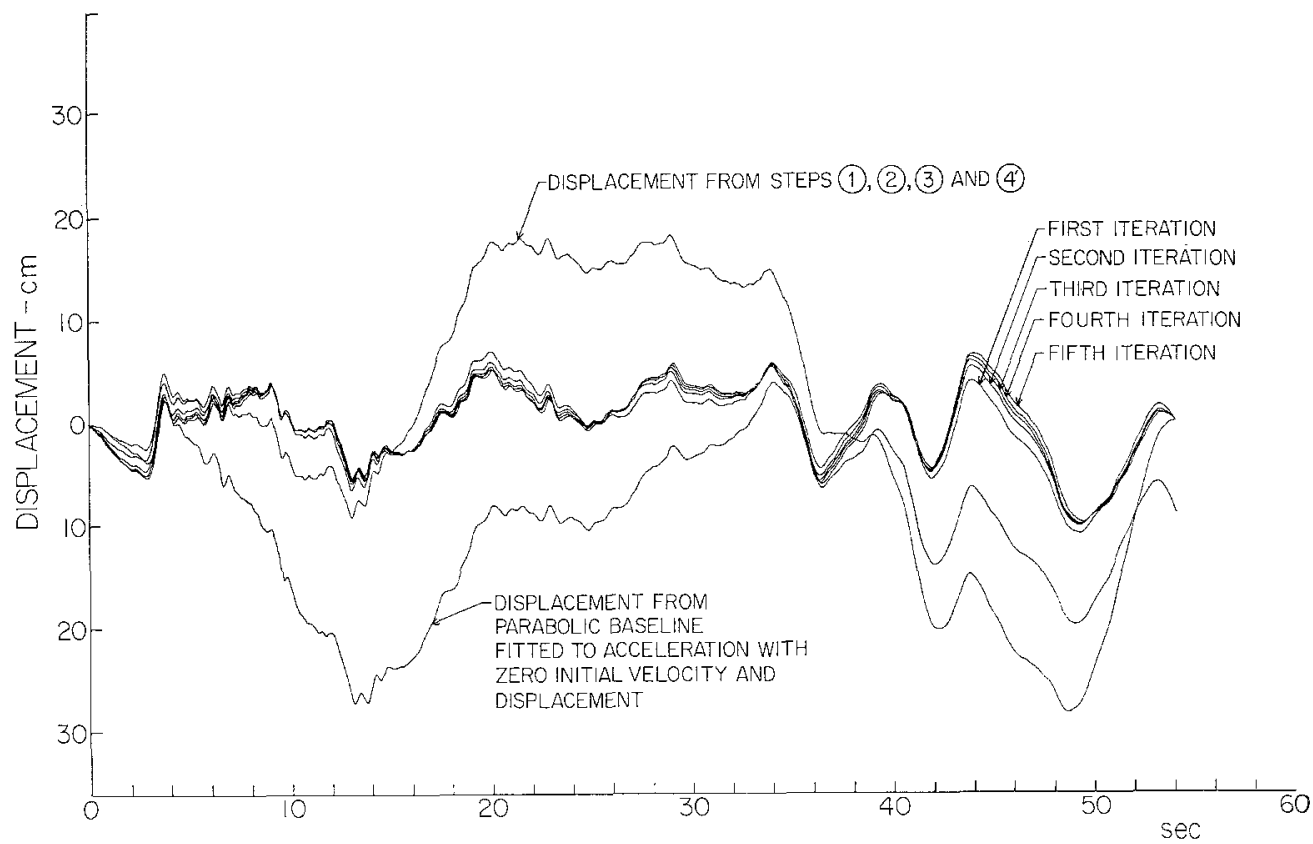

FIg. 6. A comparison of the ground-displacement curves from high-pass filtered and parabolic baseline-corrected Taft accelerograms.

and $4^{\prime}$ shown in Figure 1. This ground displacement derived from the uncorrected data, prior to any filtering, contains long-period random fluctuations introduced by digitization. When the accelerogram $a_{1}\left(t_{i}\right)$ is now processed once through the standard filtering procedure following steps $5,6, \cdots, 14,15$ shown in Figure 1, the resulting displacement is shown in Figure 6 . We shall call this sequence of processing steps $5,6, \cdots, 14$, 15 the "First Iteration." Likewise we will call steps $16,6, \cdots, 14,15$ the "Second Iteration" and so on (see Figure 1).

As described above, the fact that step 3 (Figure 1) is performed on the unfiltered 
data may cause small changes in the new values of $v_{1}$ (step 12) obtained upon subtracting the low-pass filtered accelerogram base line. A change in $v_{1}$ in turn affects the low-pass filtered base line by a small amount. By performing several iterations, these changes can be made sufficiently small so that their effect on the integrated ground displacement is negligible. To illustrate this, five iterations were performed for the Taft record (Figure 5) and are shown in Figure 6. As may be seen, the changes in the computed ground displacement from the first to fifth interation are small. The values $v_{0}$ and $v_{1}$ at the end of each iteration (after step 12, Figure 1) are shown in Table 1.

TABLE 1

VALUES For $v_{0}$ AND $v_{1}$ AT THE END OF EACH of Five Iterations

\begin{tabular}{ccc}
\hline Iteration No. & $v_{0}(\mathrm{~cm} / \mathrm{sec})$ & $v_{1}\left(\mathrm{~cm}^{2} / \mathrm{sec}^{2}\right)$ \\
\hline 1 & 1.07 & 0.00639 \\
2 & 1.59 & 0.00525 \\
3 & 1.74 & 0.00488 \\
4 & 1.77 & 0.00463 \\
5 & 1.75 & 0.00443 \\
\hline
\end{tabular}

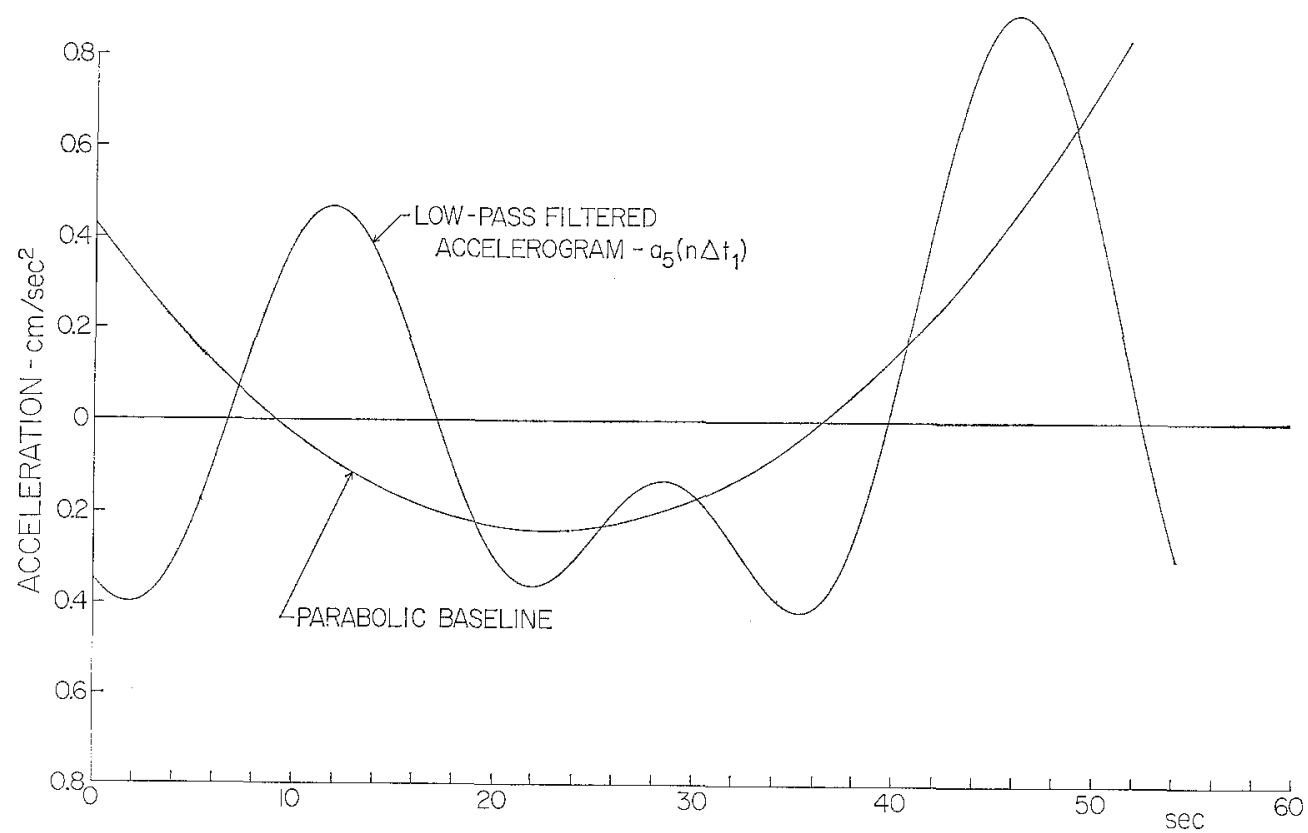

Fig. 7. A comparison of the low-pass filtered Taft accelerogram and the parabolic base line.

It may be noted here that the resolution of the standard uncorrected accelerograms is $0.1 \mathrm{~cm} / \mathrm{sec}^{2}$. Thus, the changes in $v_{1}$ after even the first iteration are much smaller than the least significant figure in the uncorrected accelerogram data. On the basis of these small changes which were also studied for several other accelerograms, we suggest that the standard baseline correction should consist of only one iteration.

In order to compare the above-outlined procedure and the classical parabolic baseline correction, we have performed this correction on the uncorrected accelerogram data and plotted the resulting ground displacement in Figure 6. This comparison clearly shows the advantages of the filtering methods over the parabolic baseline correction 
for long-record lengths. For shorter-record lengths, however, the parabolic baseline correction would yield displacements very similar to those obtained by the filtering approach.

Figure 7 shows the low-pass filtered accelerogram $a_{5}\left(n \Delta t_{1}\right)$ (Figure 1) during the first iteration. This is in effect the base line assumed in the above-described filtering procedure. For comparison, the parabolic base line is given in the same figure.

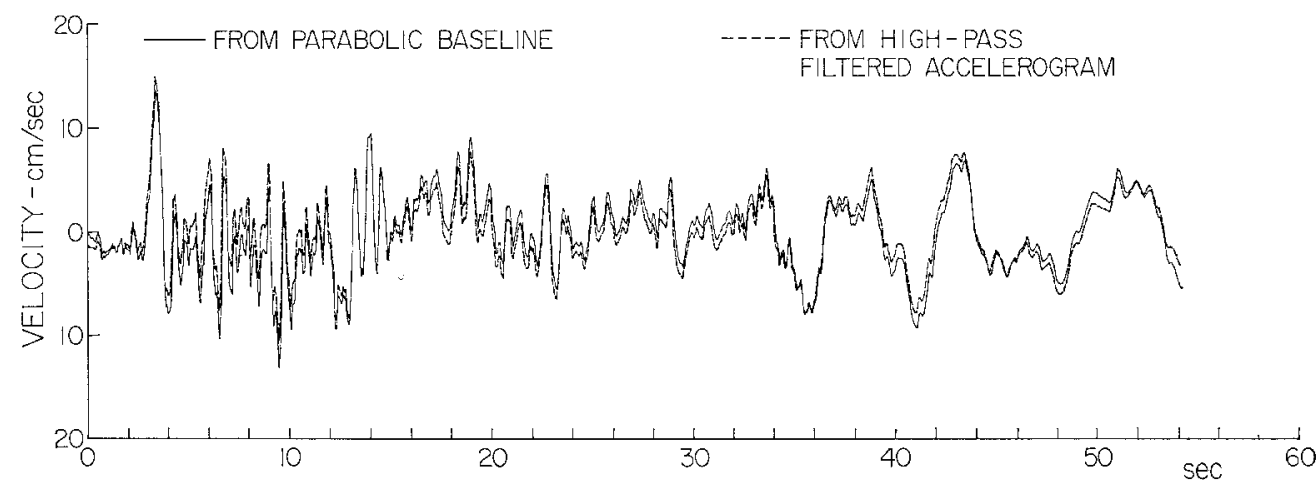

Frg. 8. A comparison of the ground-velocity curves from high-pass filtered and parabolic baseline-corrected Taft accelerograms.

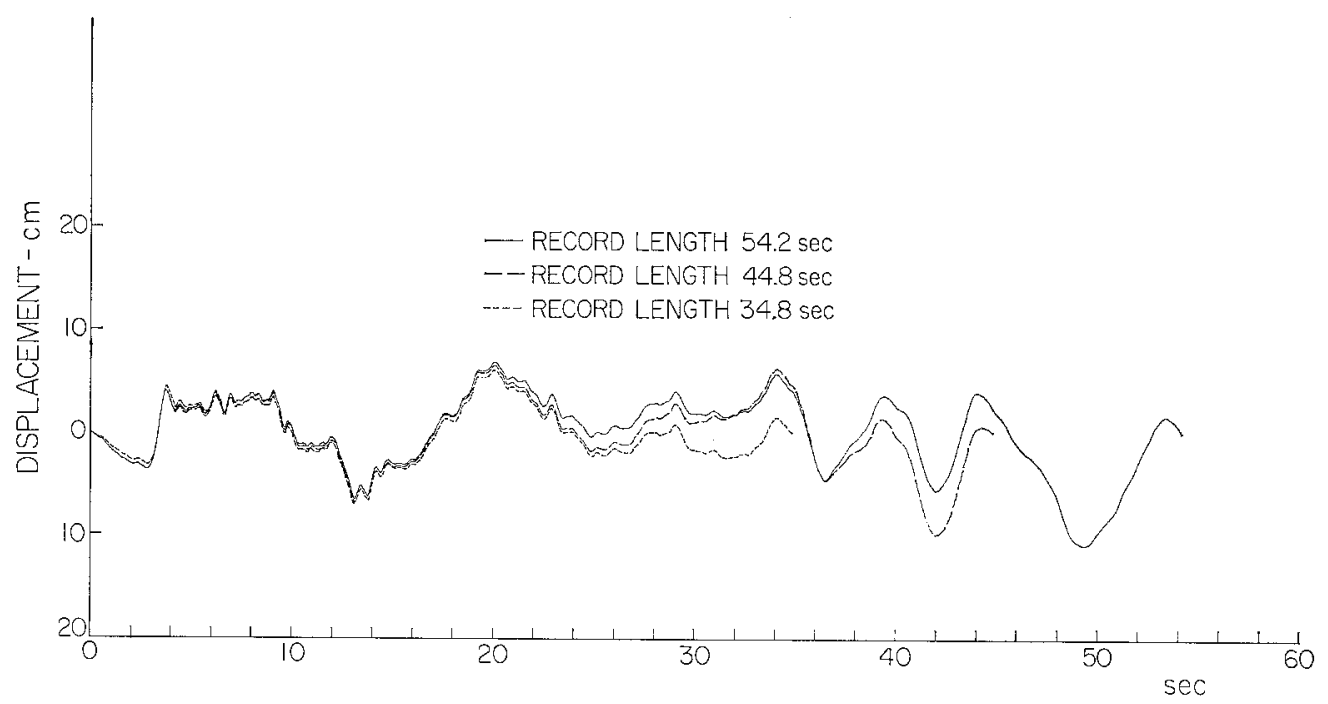

FIG. 9. The effect of record length for the new method of baseline correction of Taft accelerograms.

Marked differences in the resulting ground displacement from the filtering method and the parabolic baseline method are caused by the long-period components in the corrected accelerogram emphasized by double integration. The apparent discrepancies are considerably diminished when ground-velocity curves are compared for the two methods of baseline correction (Figure 8).

To demonstrate the degree of change in the resulting displacement curves as a function of the record length, for the high-pass filtered accelerogram, the first iteration was performed for the Taft record (Figure 5) and for the durations of about 55, 45, and 35 sec. After double integration (step 15, Figure 1), ground-displacement curves appear 
as in Figure 9. Constants $v_{0}$ and $v_{1}$ are shown in Table 2. As may be seen in Figure 9, the change in the resulting displacement curves as a function of the record length is small and can be mainly attributed to the changes in $v_{0}$ and $v_{1}$.

In closing, it should be mentioned that the choice of $\Delta t=0.02$ and $\Delta t_{1}=0.2 \mathrm{sec}$ as well as that of $f_{T}-f_{C}=0.02 \mathrm{cps}$ is somewhat arbitrary and could be slightly changed without significant altering of the properties of the resulting digital filters. It is believed that the above values allow sufficient numerical accuracy and do not lead to long machine execution time so that they are acceptable for the standard baseline correction.

TABLE 2

Constants $v_{0}$ AND $v_{1}$

\begin{tabular}{ccc}
\hline Record Duration & $v_{0}(\mathrm{~cm} / \mathrm{sec})$ & $v_{1}\left(\mathrm{~cm} / \mathrm{sec}^{2}\right)$ \\
\hline $55 \mathrm{sec}$ & 1.07 & 0.00639 \\
$45 \mathrm{sec}$ & 1.02 & 0.00629 \\
$35 \mathrm{sec}$ & 0.565 & 0.0167 \\
\hline
\end{tabular}

\section{Conclussions}

Although no physical justification may be given for a zero baseline correction curve of higher order than the first, it has been generally agreed that the classical parabolic baseline correction successfully removes some short and intermediately long-period errors in the digitized accelerograms of short duration. These errors are mainly caused by warping of the recording paper and other similar distortions. It is also well known that this correction does not seriously affect the accuracy of the response spectra calculations for periods up to about 5 sec.

The old parabolic correction may still be used for many future applications when long-period effects of accelerograms do not play an important role, but it does not meet all of the requirements for the standard baseline correction, because it depends on the record length. However, the present and future applications in structural analysis and earthquake mechanism studies require that the accuracy of the digitized, baseline-corrected accelerograms be extended to longer periods, for which the parabolic base line is not adequate.

A new method for standard baseline correction is proposed, which is based on wellknown digital filtering methods. The corrected accelerograms are the result of highpass filtering of the uncorrected data. Unlike the parabolic baseline correction, the new method is largely independent of the record length and has a well-defined frequency transfer function.

Finally, it may be noted that any permanent ground displacements are eliminated or distorted by all baseline correction procedures. The new method presented here eliminates long-period and d.c. ground motion by filtering long-period Fourier components longer than about 16 sec. Since there is no data processing method that can give accurate permanent ground displacements derived from recorded accelerograms, other methods of recording and other evidence must be used to infer such information.

\section{Acknowledgments}

I am indebted to Professors D. E. Hudson and G. W. Housner, Dr. A. G. Brady, and Mr. F. E. Udwadia for critically reading the manuscript and offering many valuable suggestions.

This study was supported by the National Science Foundation Grant GK 1197X (Engineering Mechanics Program) while the author was at the California Institute of Technology. The work was completed at the Lamont-Doherty Geological Observatory of Columbia University. 


\section{REFERENCES}

Amin, M. and A. H. S. Ang (1966). A nonstationary stochastic model for strong-motion earthquakes, Structural Research Series No. 306, University of Illinois, Urbana.

Berg, G. V. and G. W. Housner (1961). Integrated velocity and displacement of strong earthquake ground motion. Bull Seism. Soc. Am. 51, 175-189.

Berg, G. V. (1963). A study of errors in response spectrum analysis, Primera Jornadas Chilenas de Sismologia e Ingenieria Antisismica, Asociacion Chilena de Sismologia e Ingenieria Antisismica, Santiago.

Boyce, W. H. (1970). Integration of Accelerograms, Bull. Seism. Soc. Am. 60, 261-263.

Brady, A. G. (1966). Studies of Response to Earthquake Ground Motion, Earthquake Engineering Research Laboratory, California Institute of Technology, Pasadena.

Cloud, W. K. (1964). Instruments for Earthquake Analysis, Earthquake Investigations in the Western United States, 1931-1964, D. S. Carder, Editor, Publ. 41-42, U. S. Department of Commerce, Coast and Geodetic Survey, Washington, D. C.

Fremd, V. M. (1963). Installation with Memory for the Recording of Strong Earthquakes, Trudy Institula Fiziki Zemli, no. 26, (193), Izd. AN SSR, Academy of Sciences, USSR, Moscow.

Halverson, H. T. (1965). The Strong Motion Accelerograph, Proceedings of the Third World Conference on Earihquake Engineering, New Zealand.

Halverson, H. T. (1970). Modern Trends in Strong Movement (Strong-Motion) Instrumentation, Proceedings of the Conference on Dynamic Waves in Civil Engineering, J. Wiley and Son, Sussex, England.

Hershberger, J. (1955). Recent developments in strong-motion analysis, Bull. Seism. Soc. Am. $45,11-21$.

Holloway, L. J. (1958). Smoothing and filtering of time series and space fields, Advan. Geophys.4, $351-389$

Housner, G. W. (1947). Ground displacement computed from strong-motion accelerograms, Bull. Seism. Soc. Am. 37, 299-305.

Hudson, D. E. (1963). The measurement of ground motion of destructive earthquakes, Bull. Seism. Soc. Am. 53, 419-437.

Hudson, D. E. (1965). Ground motion measurements in earthquake engineering, Proceedings of the Symposium on Earthquake Engineering, University of British Columbia, Vancouver.

Hudson, D. E., N. C. Nigam, and M. D. Trifunac (1969). Analysis of Strong-Motion Accelerograph Records, Fourth World Conference on Earthquake Engineering, Santiago, Chile.

Hudson, D. E., A. G. Brady, and M. D. Trifunac (1969). Strong Motion Earthquake Accelerograms, Digitized and Plotted Data, Vol. I, Earthquake Engineering Research Laboratory, EERL 69-20, California Institute of Technology, Pasadena.

Jenschke, V. A. and J. Penzien (1964). Ground motion accelerogram analysis including dynamical instrumental correction, Bull. Seism. Soc. Am. 54, 2087-2098.

McComb, H. E., A. C. Ruge, and F. Neumann (1943). The determination of true ground motion: A symposium, Bull. Seism. Soc. Am. 33, 1-63.

McLennan, G. A. (1969). An exact correction for accelerometer error in dynamic seismic analysis, Bull. Seism. Soc. Am. 59, 705-715.

Ormsby, J. F. A. (1961). Design of numerical filters with application to missile data processing, Assoc. Comp. Mach. J. 8, 440-466.

Poppitz, J. V. (1968). Velocity and displacement of explosion-induced earth tremors derived from acceleration., Bull. Seism. Soc. Am. 58, 1573-1582.

Schiff, A. and J. L. Bogdanoff (1967). Analysis of current methods of interpreting strong-motion accelerograms, Bull. Seism. Soc. Am. 57, 857-874.

Trifunac, M. D. and D. E. Hudson (1970). Laboratory Evaluation and Instrument Corrections of Strong-Motion Accelerographs, Earthquake Engineering Research Laboratory, EERL 70-04, California Institute of Technology, Pasadena.

Trifunac, M. D. (1970). Low Frequency Errors and a New Method for Zero Baseline Correction of Strong-Motion Accelerograms., Earthquake Engineering Research Laboratory, EERL 70-07, California Institute of Technology, Pasadena.

Drvision of Engineering ANd Applied SCIEnce

Caltfornia Institute of Technology

Pasadena, California, 91109

Manuseript received January 6, 1971. 